ARTICLE

\title{
Metabotropic GABA signalling modulates longevity in C. elegans
}

Lei Chun ${ }^{1,2}$, Jianke Gong ${ }^{1,2}$, Fengling Yuan ${ }^{1}$, Bi Zhang ${ }^{1,2}$, Hongkang Liu' ${ }^{1}$, Tianlin Zheng ${ }^{1}$, Teng Yu ${ }^{1}$, X.Z. Shawn Xu ${ }^{2}$ \& Jianfeng Liu ${ }^{1}$

The nervous system plays an important but poorly understood role in modulating longevity. GABA, a prominent inhibitory neurotransmitter, is best known to regulate nervous system function and behaviour in diverse organisms. Whether GABA signalling affects aging, however, has not been explored. Here we examined mutants lacking each of the major neurotransmitters in C. elegans, and find that deficiency in GABA signalling extends lifespan. This pro-longevity effect is mediated by the metabotropic $G A B A_{B}$ receptor $G B B-1$, but not ionotropic $G_{A B A}$ receptors. GBB-1 regulates lifespan through $G$ protein-PLC $\beta$ signalling, which transmits longevity signals to the transcription factor DAF-16/FOXO, a key regulator of lifespan. Mammalian $\mathrm{GABA}_{B}$ receptors can functionally substitute for GBB-1 in lifespan control in $C$. elegans. Our results uncover a new role of GABA signalling in lifespan regulation in $C$. elegans, raising the possibility that a similar process may occur in other organisms.

\footnotetext{
${ }^{1}$ College of Life Science and Technology, Collaborative Innovation Center for Brain Science, Huazhong University of Science and Technology, Wuhan, Hubei 430074, China. ${ }^{2}$ Department of Molecular and Integrative Physiology, Life Sciences Institute, University of Michigan, Ann Arbor, Michigan 48109, USA. Correspondence and requests for materials should be addressed to J.L. (email: jfliu@mail.hust.edu.cn) or to X.Z.S.X. (email: shawnxu@umich.edu).
} 
A ging is a complex physiological process modulated by a multitude of genetic and environmental factors ${ }^{1,2}$. Recent work has revealed an important role of the nervous system in modulating aging ${ }^{1,3,4}$. For example, in insulin/IGF-1 signalling, one of the best characterized longevity-regulatory pathways, the DAF-2 insulin/IGF-1 receptor primarily acts in the nervous system to regulate lifespan in Caenorhabditis elegans ${ }^{5}$. Interestingly, work in mice revealed a similar mechanism ${ }^{6}$. Environmental factors also affect aging by impinging on the nervous system $1,3,4,7,8$. Apparently, neurons can act cell nonautonomously to modulate aging, and neuroendocrine signalling plays an important role in this process ${ }^{1,3,4,7,8}$. However, whether and how neurotransmitter signalling modulates aging is poorly understood.

GABA is the primary inhibitory neurotransmitter in the mammalian brain ${ }^{9,10}$. It is critical for the function and development of the nervous system and plays a key role in behavioural control ${ }^{11-14}$. GABA acts through both ionotropic and metabotropic receptors ${ }^{15,16}$. As ion channels, ionotropic $\mathrm{GABA}_{\mathrm{A}}$ receptors mediate the acute, fast actions of GABA ${ }^{17,18}$. By contrast, metabotropic $G_{A B A}$ receptors are $G$ protein coupled receptors (GPCRs) that execute the slow, long-lasting effects of GABA ${ }^{19-22}$.

C. elegans is a popular model organism widely utilized to study the biology of aging, featuring a short generation time and lifespan, as well as conserved genetic mechanisms that regulate longevity ${ }^{1,2}$. C. elegans has also been widely used as a genetic model for neurobiology ${ }^{23}$. Although C. elegans possesses a relatively small nervous system, it produces all the major neurotransmitters (for example, $\mathrm{ACh}$, glutamate, GABA and biogenic amines) and their cognate receptors, all of which are encoded by evolutionarily conserved gene families $^{23}$. While the role of neurotransmitters in controlling behaviour and nervous system development and function has been extensively characterized, little is known about their role in aging.

Here we interrogated the role of neurotransmitters in lifespan control in C. elegans and found that GABA regulates lifespan through $G_{A B A}$ receptor signalling. We further identified a genetic pathway that cell nonautonomously transmits longevity signals from the $\mathrm{GABA}_{\mathrm{B}}$ receptor in motor neurons to the transcription factor FOXO/DAF-16 in the intestine. Our results identify a novel function of GABA beyond its conventional role in modulating animal behaviour and nervous system function and development.

\section{Results}

Deficiency in GABA signalling extends lifespan. The nervous system of $C$. elegans hermaphrodites is relatively simple ${ }^{23,24}$, making it convenient to characterize the role of neurotransmission in lifespan control. We first assayed the lifespan of unc-13 mutant worms that are defective in synaptic transmission ${ }^{25,26}$. These mutant animals are long lived (Fig. 1a and Supplementary Table 1), consistent with previous results ${ }^{27}$. The fact that unc-13 mutant animals are long-lived indicates that deficiency in neurotransmitter signalling extends lifespan. To ascertain which neurotransmitter(s) may contribute to this effect, we examined mutant animals defective in each of the major neurotransmitters, including unc-17 (ACh), eat-4 (glutamate), cat-2 (dopamine), tph-1 (serotonin), tdc-1 (tyramine), tbh-1 (octopamine) and unc-25 (GABA) ${ }^{28-33}$ (Fig. 1b-h). Among these
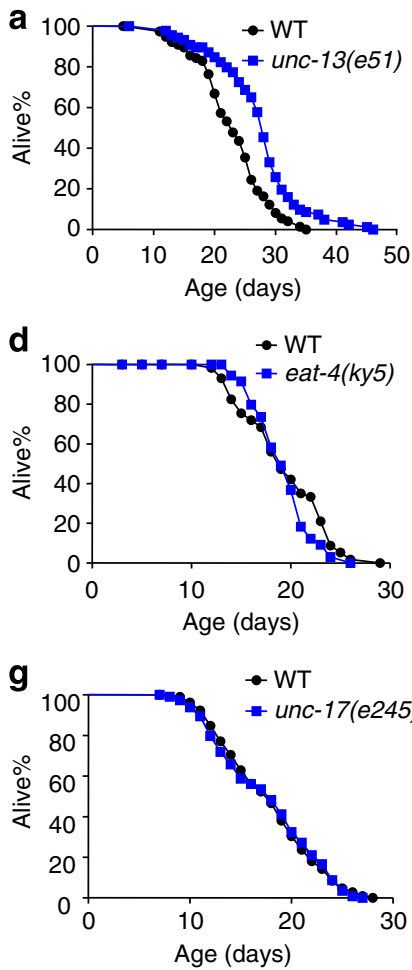

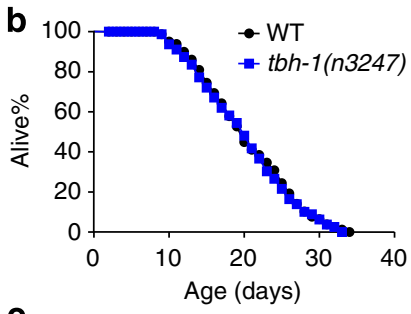

e
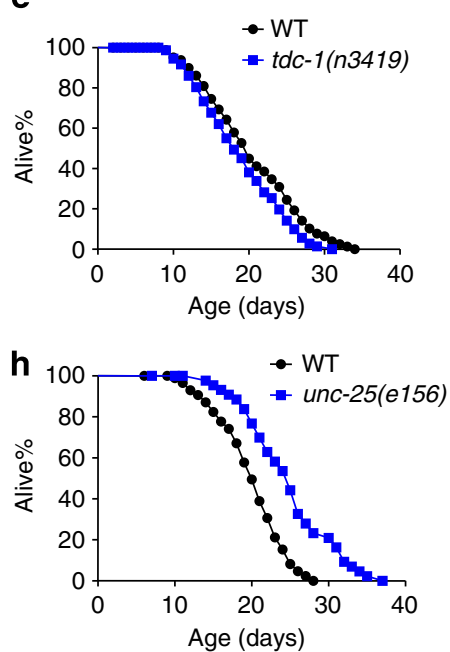
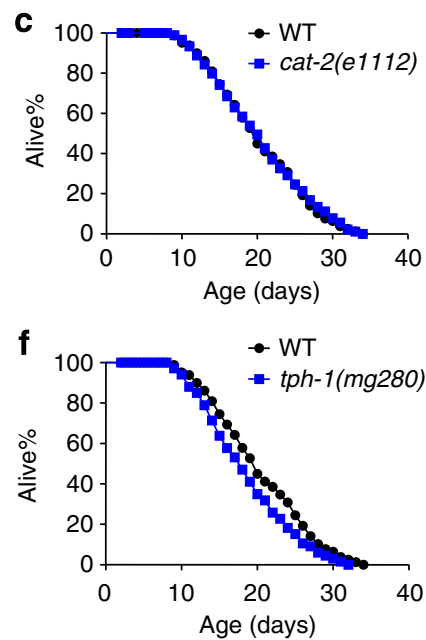
mutants, unc-25 worms lived the longest life, showing the most pronounced phenotype (Fig. 1h), while others only had modest or no effect (Fig. 1b-g). While these data by no means exclude a role of other neurotransmitters, it reveals an important function of GABA signalling in lifespan control.

Loss of the $\mathrm{GABA}_{\mathrm{B}}$ receptor gene $\boldsymbol{g} \boldsymbol{b} \boldsymbol{b}$-1 extends lifespan. GABA acts through both ionotropic and metabotropic receptors. The $C$. elegans genome encodes two metabotropic $\mathrm{GABA}_{\mathrm{B}}$ receptor genes, $g b b-1$ and $g b b-2$, which are highly homologous to their mammalian counterparts ${ }^{34}$. We found that $g b b-1$ but not $g b b-2$ mutants were long-lived (Fig. 2a). $g b b-1 ; g b b-2$ double mutant was indistinguishable from $g b b-1$ single mutant (Fig. 2a), indicating a specific role of $g b b-1$ in regulating lifespan. By contrast, mutant worms lacking unc-49, which encodes the sole evolutionarily conserved $\mathrm{GABA}_{\mathrm{A}}$ receptor family member ${ }^{35}$, were normally lived (Fig. 2b). These results identify a role for the $\mathrm{GABA}_{\mathrm{B}}$ receptor gene $g b b-1$ in lifespan control.

$g b b-1$ is enriched in the nervous system but is also found to be expressed in other tissues such as muscle and intestine ${ }^{34}$. We therefore asked where $g b b-1$ acts to regulate lifespan. Using tissue-specific promoters, we found that expression of $g b b-1$ cDNA in neurons, but not in muscle or intestine, rescued $g b b-1$ lifespan phenotype (Fig. 3a). Thus, gbb-1 appears to act in neurons to regulate lifespan.

We crossed the $g b b-1$ neuronal transgene into wild-type background and found that it rendered worms short-lived (Fig. 3b). Thus, overexpression of $g b b-1$ shortens lifespan. This is consistent with our mutant data that loss of $g b b-1$ extends lifespan, providing further evidence supporting that $g b b-1$ regulates longevity.

GBB-1-dependent lifespan regulation requires DAF-16/FOXO. We wondered how $g b b-1$ controls lifespan. Various genetic and environmental factors regulate lifespan by converging on a subset of transcription factors ${ }^{1}$. We therefore examined the major transcription factors known to regulate lifespan, and found that RNAi of daf-16 completely suppressed the lifespanextending phenotype of $g b b-1$ mutant worms (Fig. 4a), while RNAi of other transcription factors such as hsf-1, skn-1 and pha-4 did not (Fig. $4 \mathrm{~b}-\mathrm{d}$ ). Thus, daf-16 is required for the function of $g b b-1$, suggesting that $g b b-1$ signals to daf-16 to regulate lifespan.

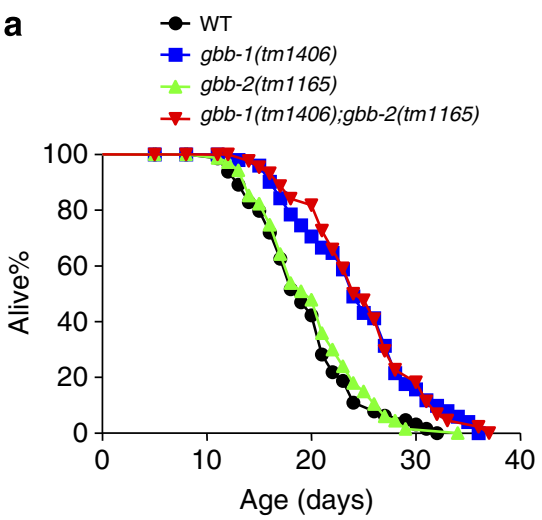

Gi/o-PLC $\beta$ transmits longevity signals from GBB-1 to DAF-16. DAF-16 is best known to act downstream of insulin/IGF-1 signalling to regulate lifespan. Does GBB-1 genetically interact with insulin/IGF-1 signalling? To test this, we assayed the lifespan of $g b b$-1;daf-2 double mutant. daf-2 encodes the sole worm insulin/ IGF-1 receptor homologue ${ }^{36}$. The double mutant exhibited a lifespan significantly longer than both $g b b-1$ and $d a f-2$ single mutants (Supplementary Fig. 1), suggesting that $g b b-1$ and daf-2 may act in different pathways. However, as a reduction-offunction allele of $d a f-2$ was used here (daf-2 nulls are lethal), we did not further test this model. Thus, we do not rule out the possibility that $g b b-1$ and $d a f-2$ may act in the same or overlapping pathways.

Then how does GBB-1 signal to DAF-16? As a GPCR, GBB-1 is unlikely to communicate with DAF-16 directly. We then sought to identify genes that transduce longevity signals from GBB-1 to DAF-16. As the $\mathrm{GABA}_{\mathrm{B}}$ receptor is known to be coupled to $\mathrm{Gi} / \mathrm{o}$ PLC $\beta$ signalling ${ }^{10,37}$, we examined this pathway. The worm genome encodes one Go orthologue, goa-1 (refs 38,39), and at least half a dozen Gi-related genes ${ }^{40}$. However, unlike $g b b-1$, goa1 mutant worms are not long-lived (Supplementary Fig. 2), suggesting the presence of functional redundancy from other $\mathrm{Gi} / \mathrm{o}$ genes. To overcome this difficulty, we attempted to block Gi/o signalling using PTX (Pertussis toxin) by expressing it as a transgene in worm neurons. We found that inactivation of $\mathrm{Gi} / \mathrm{o}$ by PTX extended lifespan (Fig. 5a). This PTX-dependent lifespan extension also required daf-16, as it can be blocked by daf-16 RNAi (Fig. 5a), suggesting that Gi/o may act upstream of DAF-16 to regulate lifespan. Further, the PTX transgene also suppressed the short-lived phenotype caused by overexpression of $g b b-1$ (Fig. 5b), suggesting that Gi/o acts downstream of $g b b-1$. These data support our model that Gi/o acts downstream of GBB-1 but upstream of DAF-16.

The $\mathrm{GABA}_{\mathrm{B}}$ receptor is known to pass signals from $\mathrm{Gi} / \mathrm{o}$ heterotrimeric $G$ proteins to PLC $\beta$ (refs 10,37). The worm genome encodes one PLC $\beta$ homologue, EGL-8 (refs 41,42). Similar to $g b b-1$ mutant and PTX-transgenic worms, egl-8 mutant worms were long-lived (Fig. 5c), consistent with the model that egl-8 acts in the pathway. Importantly, the long-lived phenotype associated with egl-8 mutant worms was suppressed by daf-16 (Fig. 5c), suggesting that egl-8 acts upstream of daf-16. Furthermore, loss of egl-8 suppressed the short-lived phenotype caused by overexpression of $g b b-1$ (Fig. $5 \mathrm{~d}$ ), suggesting that egl- 8 acts downstream of $g b b-1$. A prior study also showed that

b

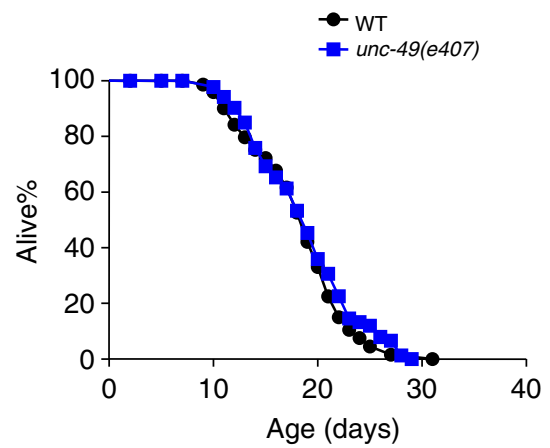

Figure 2 | Loss of the $\mathbf{G A B A}_{\mathbf{B}}$ receptor gene $\mathbf{g} \boldsymbol{b} \mathbf{b}-\mathbf{1}$ extends lifespan. (a) gbb-1 mutant worms are long-lived, but gbb-2 mutant worms are not (log-rank test, $P<0.001, g b b-1$ mutant worms compared with wild type; $P=0.518, g b b-2$ mutant worms compared with wild type; $P<0.001, g b b-1 ; g b b-2$ mutant worms compared with wild type. $n=44-67$ for different genotypes). (b) unc-49 mutant worms show normal lifespan (log-rank test, $P=0.353, n=67-76$ for different genotypes). All lifespan assays were carried out at $20^{\circ} \mathrm{C}$ and were repeated at least three times. Please see Supplementary Table 1 for detailed statistical analysis of lifespan data. 

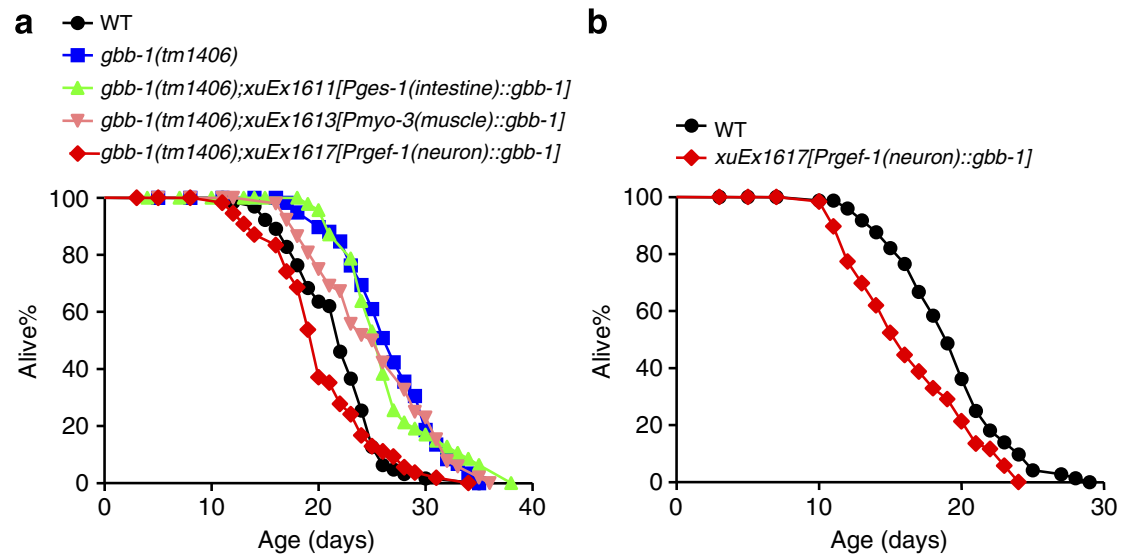

Figure $3 \mathbf{~ g ~} \mathbf{b} b-1$ acts in neurons to regulate lifespan. (a) Transgenic expression of $g b b-1$ in neurons (log-rank test, $P=0.198$ ), but not in the intestine (logrank test, $P<0.001$ ) or muscles (log-rank test, $P<0.001$ ), suppresses the long-lived phenotype of $g b b-1$ mutant worms (log-rank test, $P<0.001$ ). The rgef- 1 , ges-1 and myo-3 promoters were used to drive gbb-1 cDNA expression in neurons, intestine and muscles, respectively ${ }^{61-63}$ ( $n=47-63$ for different genotypes). (b) Overexpression of $g b b-1$ cDNA in neurons shortens lifespan (log-rank test, $P=0.001, n=53-72$ for different genotypes). Each plasmid DNA listed above was injected at a concentration of $50 \mathrm{ng} \mu \mathrm{l}^{-1}$. All lifespan assays were carried out at $20^{\circ} \mathrm{C}$ and were repeated at least three times. Please see Supplementary Table 1 for detailed statistical analysis of lifespan data.
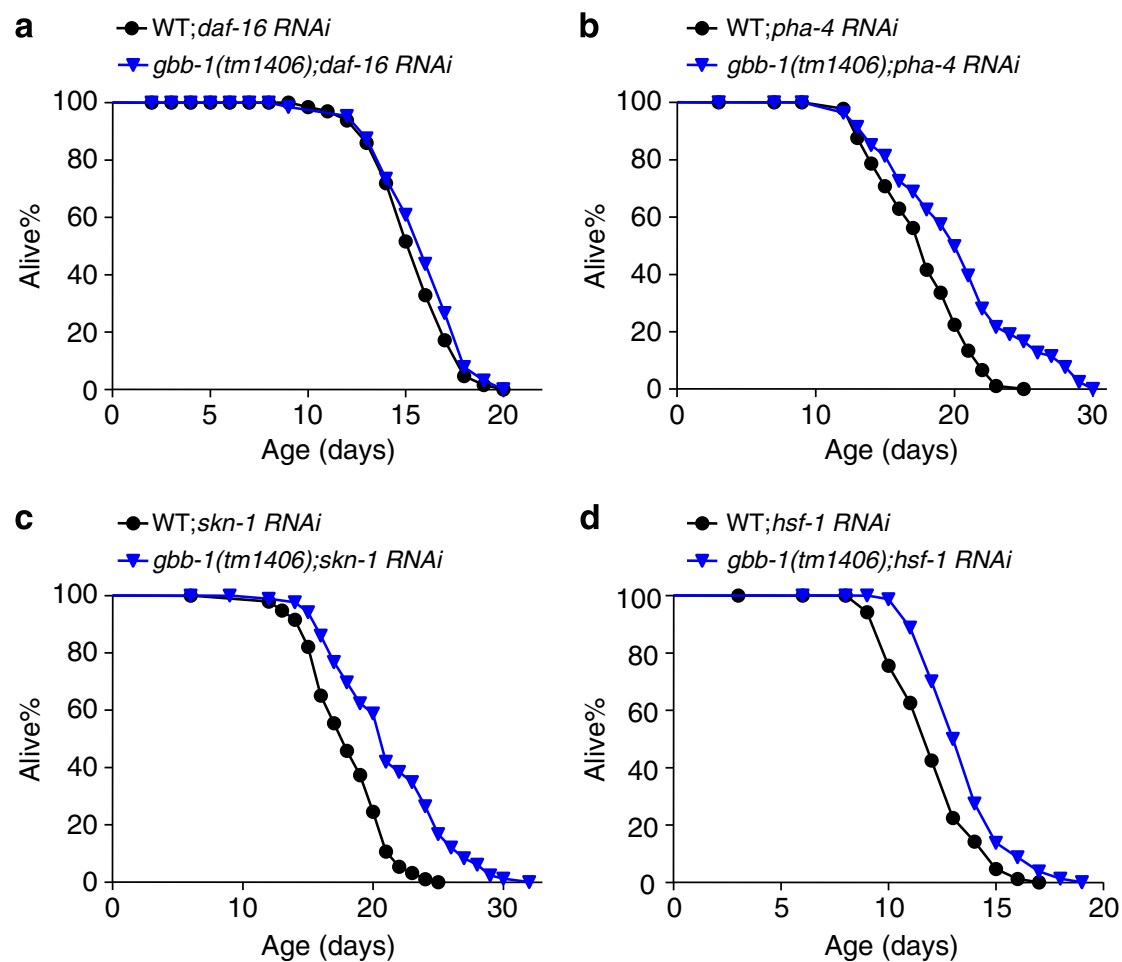

Figure 4 | GBB-1-dependent lifespan regulation requires the FOXO transcription factor DAF-16. (a) daf-16 RNAi fully suppresses the long-live phenotype of gbb-1 mutant worms (log-rank test, $P=0.502, n=78-91$ for different genotypes). (b-d) RNAi of pha-4 (b, log-rank test, $P<0.001)$, skn-1 (c, log-rank test, $P<0.001)$ or $h s f-1(\mathbf{d}$, log-rank test, $P<0.001)$ fail to suppress the long-lived phenotype of $g b b-1$ mutant worms $(n=79-94$ for different genotypes). For RNAi experiments, NGM plates included carbenicillin $\left(25 \mu \mathrm{g} \mathrm{ml}^{-1}\right)$ and IPTG $(1 \mathrm{mM})$. HT115 bacteria-carrying vector or RNAi plasmid were seeded on RNAi plates 2 days before the experiment. Worms were fed RNAi bacteria from the egg stage. All RNAi lifespan assays were carried out at $20^{\circ} \mathrm{C}$, and were repeated at least three times. Please see Supplementary Table 1 for detailed statistical analysis of lifespan data.

EGL-8 regulates lifespan in a DAF-16-dependent manner ${ }^{43}$. These results together support the model that $G$ proteinPLC $\beta$ signalling transduces longevity signals from GBB-1 to DAF-16.

DKF-2/PKD acts upstream of DAF-16 to regulate lifespan. DAF-16 is best known to be regulated by kinases ${ }^{1}$. We next set out to identify kinases that act downstream of PLC $\beta$ but upstream of DAF-16. Protein kinase C (PKC), a group of kinases activated by DAG and/or $\mathrm{Ca}^{2+}$, is a primary downstream target of PLC $\beta$. The worm genome encodes four PKC homologues: tpa-1, pkc-1, $p k c-2$ and $p k c-3$. However, none of these genes, when mutated or knocked down, gave rise to a long-lived phenotype as did egl-8 and $g b b-1$ (Supplementary Fig. 3a-d). These data suggest that PKC is unlikely to mediate the effect of EGL-8/PLC $\beta$ in longevity signalling. 

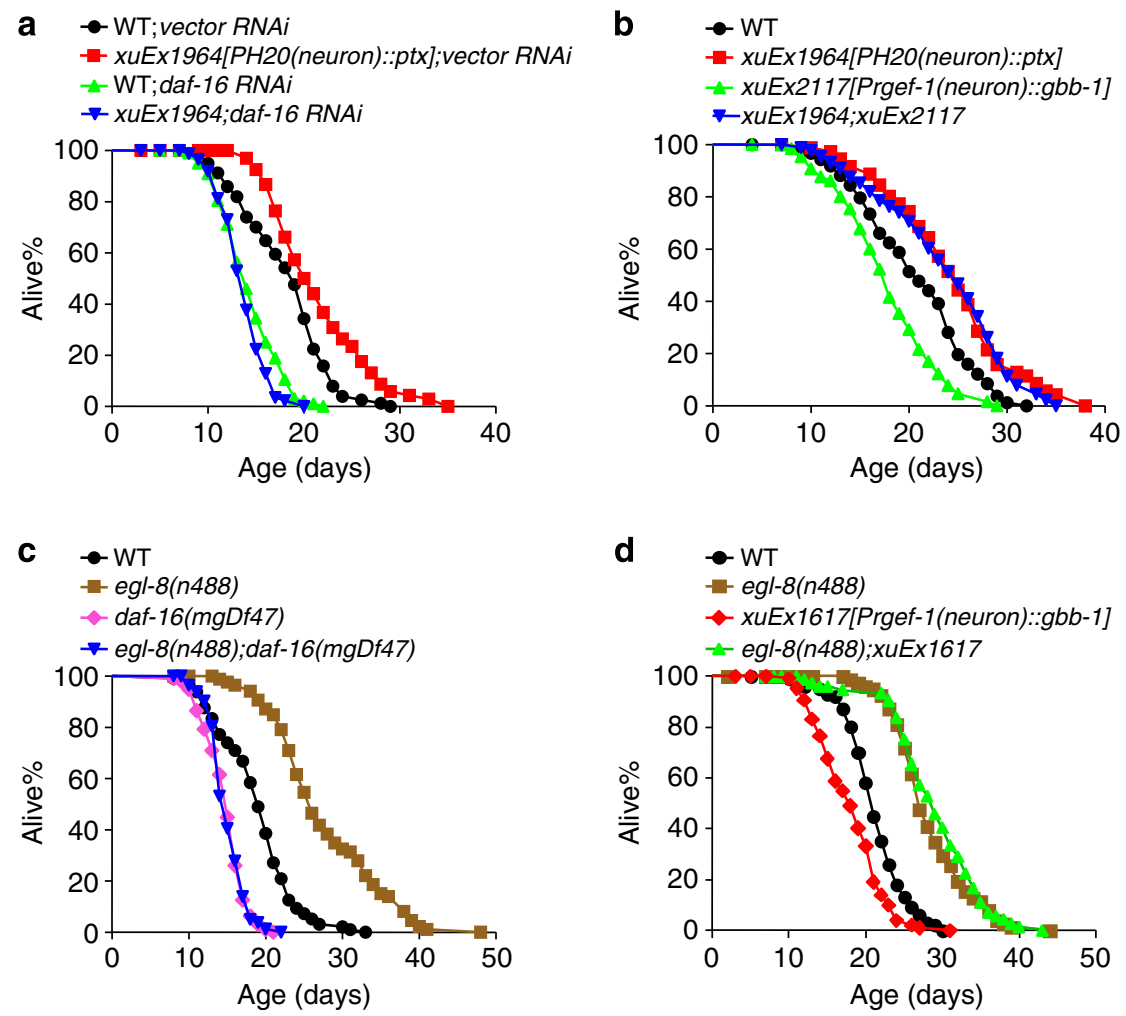

Figure 5 | G protein-PLC $\beta$ signalling transmits longevity signals from GBB-1 to DAF-16. (a) Inhibition G protein function by transgenic expression of PTX in neurons extends lifespan (log-rank test, $P<0.001$ ), which can be fully suppressed by daf-16 RNAi (log-rank test, $P=0.046$ ). The H2O promoter drives expression of PTX in neurons ${ }^{64}$ ( $n=68-96$ for different genotypes). (b) Transgenic expression of PTX fully suppresses the short-lived phenotype caused by $g b b-1$ transgene (log-rank test, $P<0.001$, xuEx2117 compared with the wild type; $P=0.56$, xuEx1964;xuEx2117 compared with $x u E x 1964$. $n=65-88$ for different genotypes). (c) Loss of egl-8 extends lifespan, which can by fully suppressed by daf-16(mgDf47) mutation (log-rank test, $P<0.001$, egl-8 mutant worms compared with the wild type; $P=0.781$, egl-8; daf- 16 mutant worms compared with daf-16 mutant worms. $n=57-115$ for different genotypes).

(d) Loss of egl-8 fully suppresses the short-lived phenotype caused by gbb-1 overexpression (log-rank test, $P<0.001$, xuEx1967 compared with the wild type; $P=0.382$, egl-8;xuEx1967 compared with egl-8 mutant worms. $n=72-102$ for different genotypes). All lifespan assays were carried out at $20^{\circ} \mathrm{C}$ and were repeated at least twice. 5-Fluoro-2'-deoxyuridine (FUDR) was included in assays involving egl-8 mutant worms, which show a defect in egg laying. Please see Supplementary Table 1 for detailed statistical analysis of lifespan data.

In addition to PKC, DAG can also activate another type of kinases, protein kinase D (PKD), in both a PKC-dependent and independent manner ${ }^{44}$. Two PKD homologues are found in C. elegans: $d k f-1$ and $d k f-2$ (ref. 45). We thus considered a potential role of these two PKD genes. $d k f-1$ mutant worms were short-lived (Supplementary Fig. 3e-f), suggesting that it is probably not involved. By contrast, worms lacking $d k f-2$ were long-lived (Fig. 6a), a phenotype similar to $g b b-1$ and egl-8 mutants. A previous study also showed that loss of $d k f-2$ extends lifespan and does so by regulating DAF-16 (ref. 45). Indeed, we found that the long-lived phenotype of $d k f-2$ worms was completely suppressed by loss of daf-16 (Fig. $6 \mathrm{~b}$ ), consistent with the notion that $d k f-2$ acts upstream of $d a f-16$ to regulate lifespan.

To obtain further evidence that $d k f-2$ regulates daf-16, we examined whether loss of $d k f-2$ can promote daf-16 function. We found that the mRNA levels of DAF-16 target genes, such as $m t l-1$, sod-3 and dod-3, were upregulated in $d k f-2$ mutant background (Fig. 6c). We also assayed the protein level of SOD-3::GFP fusion, a commonly used reporter for DAF-16 activity, and found that it was upregulated in $d k f-2$ worms (Fig. 6d). Thus, $d k f-2$ appears to regulate daf-16 function, providing additional evidence supporting that DKF-2 acts upstream of DAF-16 to control lifespan.

DKF-2 acts downstream of EGL-8 to regulate lifespan. We then asked whether $d k f-2$ functions in the $g b b-1$ pathway to regulate lifespan. $d k f-2 ; g b b-1$ double mutant exhibited a lifespan similar to single mutants (Fig. 6a), suggesting that they act in the same pathway. To gather additional evidence, we first rescued $d k f-2$ long-lived phenotype by expressing wild-type $d k f-2$ cDNA as a transgene in neurons in $d k f-2$ mutant worms (Fig. 6e). In addition, this $d k f-2$ transgene modestly shortened lifespan in wildtype background (Fig. 6f and Supplementary Table 1). Importantly, this transgene suppressed the long-lived phenotype of $g b b$ 1 , as well as egl-8 mutant worms (Fig. $6 \mathrm{~g}-\mathrm{h}$ ), providing additional evidence supporting that DKF-2 acts downstream of GBB-1 and EGL-8/PLC $\beta$ to regulate lifespan.

Neuron-to-intestine signalling transmits longevity signals. Apparently, the aforementioned genetic pathway, which transmits longevity signals from GBB-1 to DAF-16, does not inform where the pathway operates. Our data showed that GBB-1 acts in neurons to regulate lifespan (Fig. 3a), yet DAF-16 is best known to function in the intestine ${ }^{46}$. This raises the question whether GBB-1 signals DAF16 through neuron-to-intestine signalling. However, DAF-16 also functions in neurons ${ }^{46}$, which confounds our data interpretation. To clarify this issue, we interrogated the site of action of DAF-16 in the GBB-1 pathway. We found that transgenic expression of daf-16 cDNA in the intestine but not in neurons rescued the lifespan phenotype of $g b b$-1;daf-16 double mutant (Fig. 7a), indicating that DAF-16 acts in the intestine in the GBB-1 pathway. 
a

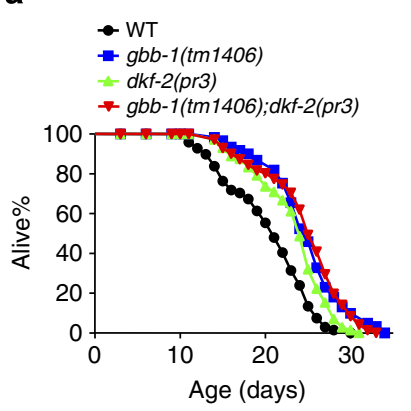

d

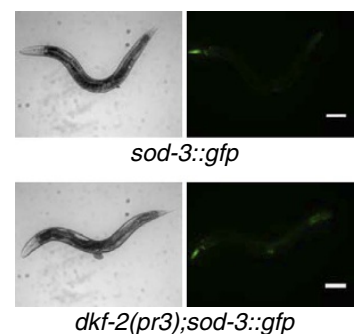

f

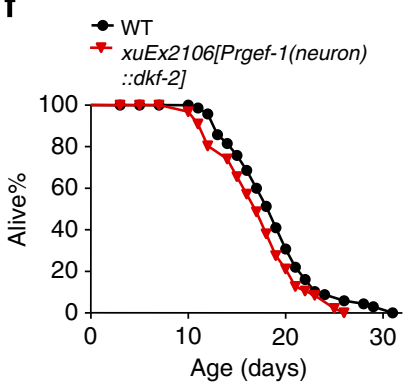

b

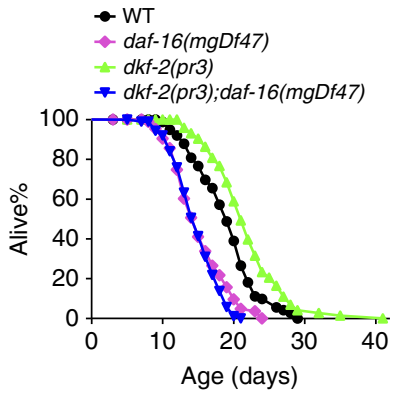

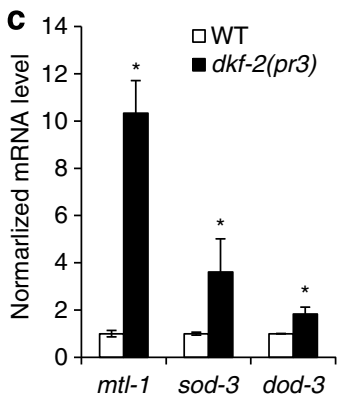

e
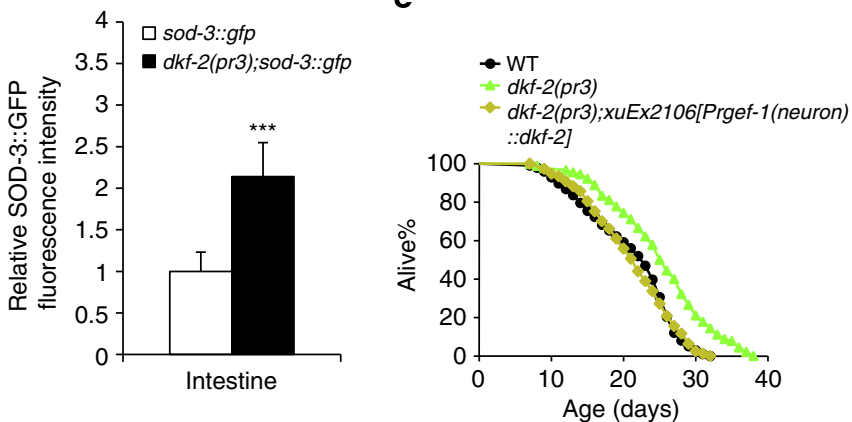

g

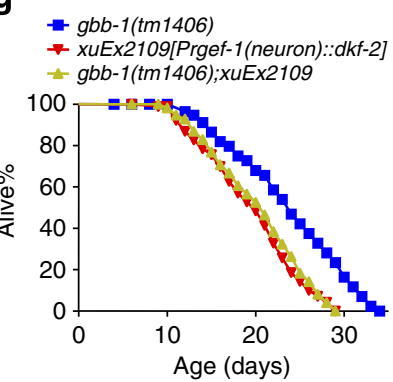

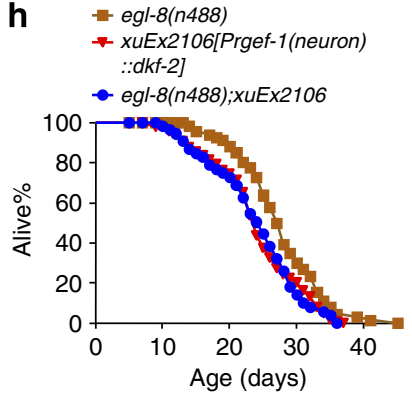

Figure 6 | The PKD homologue DKF-2 acts downstream of G protein-PLC $\beta$ signalling but upstream of DAF-16 to regulate lifespan. (a) $d k f-2$ acts in the $g b b-1$ pathway to regulate lifespan. $d k f-2$ mutant worms were long-lived (log-rank test, $P<0.001)$. $d k f-2 ; g b b-1$ double mutant showed a similar lifespan to single mutants (log-rank test, $P=0.919)$, suggesting that they are in the same pathway $(n=61-72$ for different genotypes). (b) Loss of daf-16 fully suppresses the long-lived phenotype of $d k f-2$ mutant worms (log-rank test, $P=0.114, n=72-82$ for different genotypes). (c) qPCR analysis of DAF- 16 target genes. qPCR reactions were run in triplicates for each genotype. Each experiment was repeated three times. Error bars, s.e.m. ${ }^{\star} P<0.05$ (analysis of variance (ANOVA) with Bonferroni test). (d) Quantification of SOD-3::GFP fluorescence intensity. SOD-3::GFP is encoded by the transgene muls84 (ref. 46). The left panels show representative images (selected from 10 similar images). Image quantification was performed as previously described $^{56} . n \geq 15$. Error bars, s.e.m. ${ }^{\star \star} P<0.001$ (t-test). Scale bar, $100 \mu \mathrm{m}$. (e) Overexpression of $d k f-2$ in neurons fully suppresses the long-lived phenotype of $d k f-2$ mutant worms. Prgef- 1 is a neuron-specific promoter ${ }^{62}$ (log-rank test, $P=0.143, n=77-98$ for different genotypes). (f) Overexpression of $d k f-2$ modestly shortens lifespan (log-rank test, $P=0.005, n=58-69$ for different genotypes). (g) Overexpression of $d k f-2$ fully suppresses the long-lived phenotype of $g b b-1$ mutant worms (log-rank test, $P=0.462, n=44-72$ for different genotypes). (h) Overexpression of $d k f-2$ fully suppresses the long-lived phenotype of egl-8 mutant worms (log-rank test, $P=0.967, n=50-98$ for different genotypes). All lifespan assays were carried out at $20^{\circ} \mathrm{C}$ and were repeated at least twice. FUDR was included in assays involving egl-8 mutant worms, which show a defect in egg laying. Please see Supplementary Table 1 for detailed statistical analysis of lifespan data.

GBB-1 is broadly expressed in the nervous system $^{34}$. We further asked in which groups of neurons GBB-1 regulates lifespan. Transgenic expression of $g b b-1$ cDNA in sensory neurons under the osm-6 promoter did not rescue $g b b-1$ mutant phenotype (Fig. 7c), nor did expression of $g b b-1$ in subsets of interneurons using the $g l r-1$ promoter (Fig. 7b). By contrast, restoring $g b b-1$ in ventral cord motor neurons through the acr-2 promoter fully rescued the $g b b-1$ longevity phenotype (Fig. 7d). Thus, GBB-1 can act in ventral cord motor neurons to regulate lifespan. This suggests a motor neuron-tointestine signalling axis that transmits longevity signals from GBB-1 to DAF-16.
Rat $\mathrm{GABA}_{\mathrm{B}}$ receptor can functionally substitute for GBB-1. We expressed the rat $\mathrm{GABA}_{\mathrm{B}}$ receptor, GB1/GB2 (refs 47-49), as a transgene in $g b b-1$ mutant background using a neuron-specific promoter, and found that the transgene rescued the long-lived phenotype of $g b b-1$ mutant worms (Fig. 8a). This suggests that mammalian $G_{A B A}$ receptor can functionally substitute for worm GBB-1 in regulating lifespan.

Unlike worm GBB-1, the mammalian $\mathrm{GABA}_{\mathrm{B}}$ receptor has been extensively characterized pharmacologically ${ }^{15,48,50,51}$. We thus took this advantage by testing some known antagonists of the $\mathrm{GABA}_{\mathrm{B}}$ receptor such as CGP36216 (ref. 52) and SCH50911 (ref. 53). These chemicals did not have a notable effect on lifespan 
a

WT

gbb-1(tm1406)

- daf-16(mgDf47)

$\neg$ gbb-1;daf-16

- gbb-1;daf-16;xuEx1c[Pges-1(intestine)::daf-16]

- gbb-1;daf-16;xuEx4c[Prgef-1(neuron)::daf-16]

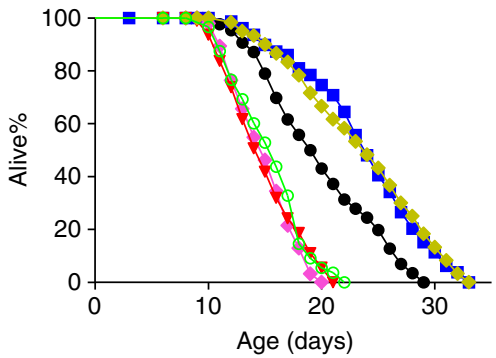

c
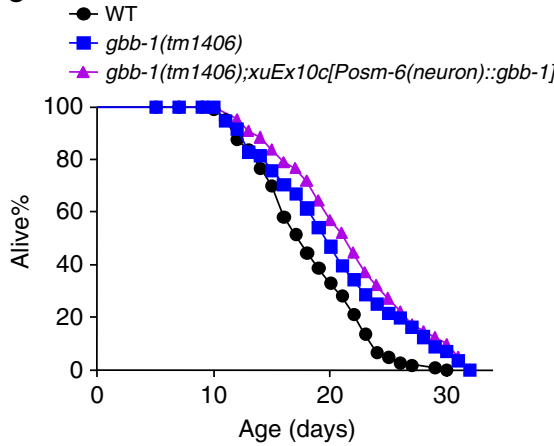

b

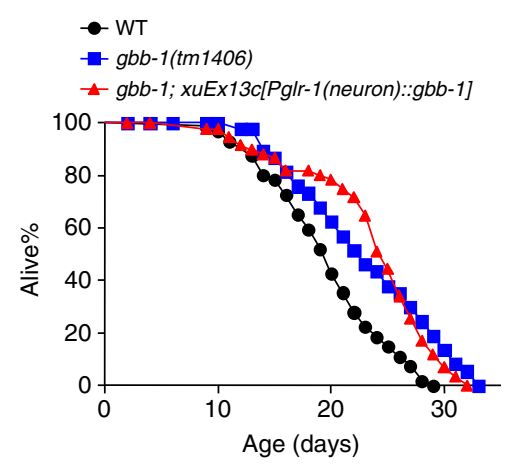

d

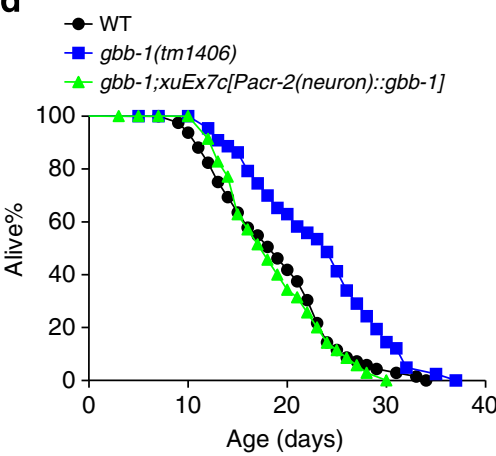

Figure 7 | GBB-1 functions in motor neurons to signal DAF-16 in the intestine to regulate lifespan. (a) Transgenic expression of daf-16 in the intestine (log-rank test, $P=0.892$ compared with gbb-1 mutant worms), but not in neurons (log-rank test, $P<0.001$ compared with gbb-1 mutant worms), suppresses the short-lived phenotype of gbb-1;daf-16 double mutant worms. The rgef-1 and ges-1 promoters were used to drive daf-16 cDNA expression in neurons and the intestine, respectively ( $n=55-93$ for different genotypes). (b) Transgenic expression of $g b b-1$ in subsets of interneurons fails to suppress the long-lived phenotype of $g b b-1$ mutant worms. The $g / r-1$ promoter was used to drive $g b b-1 \mathrm{cDNA}$ expression in interneurons (log-rank test, $P<0.001$, $n=37-60$ for different genotypes). (c) Transgenic expression of $g b b-1$ in sensory neurons fails to suppress the long-lived phenotype of $g b b-1$ mutant worms. The osm- 6 promoter was used to drive $g b b-1$ cDNA expression in sensory neurons (log-rank test, $P<0.001, n=41-103$ for different genotypes). (d) Transgenic expression of $g b b-1$ in ventral cord motor neurons fully suppresses the long-lived phenotype of gbb-1 mutant worms (log-rank test, $P=0.799, n=35-70$ for different genotypes). The acr-2 promoter was used to drive gbb-1 cDNA expression in ventral cord motor neurons. All lifespan assays were carried out at $20^{\circ} \mathrm{C}$ and were repeated at least twice. Please see Supplementary Table 1 for detailed statistical analysis of lifespan data.

in wild-type animals (Fig. 8b,c). Remarkably, when these chemicals were applied to transgenic worms expressing the rat $\mathrm{GABA}_{\mathrm{B}}$ receptor $\mathrm{GB} 1 / \mathrm{GB} 2$, they extended lifespan and did so as efficiently as $g b b-1$ mutation (Fig. $8 \mathrm{~d}, \mathrm{e}$ ). These data present further evidence supporting that the mammalian $\mathrm{GABA}_{\mathrm{B}}$ receptor can functionally substitute for worm GBB-1 in lifespan regulation, raising the possibility that it may play a similar role in mammals.

\section{Discussion}

As the primary inhibitory neurotransmitter, GABA is best known to regulate the function and development of the brain and it plays a key role in controlling animal behaviour ${ }^{11-14}$. In the current study, we identified a new role of GABA signalling in aging. Interestingly, the effect of GABA on longevity is mediated by $\mathrm{GABA}_{\mathrm{B}}$ rather than $\mathrm{GABA}_{\mathrm{A}}$ receptor. As a GPCR, GABA receptor generally meditates the slow, long-lasting actions of GABA, which is consistent with its role in regulating longevity. A previous study reported that knocking down Drosophila $\mathrm{GABA}_{\mathrm{B}}$ receptor in insulin-producing cells shortens lifespan ${ }^{54}$, an effect opposite to that observed in C. elegans. However, unlike extended lifespan, it is difficult to interpret a short-lived phenotype. As such, it remains unclear whether $\mathrm{GABA}$ and $\mathrm{GABA}_{\mathrm{B}}$ receptor modulate aging in this organism. On the other hand, mammalian $\mathrm{GABA}_{\mathrm{B}}$ receptor can functionally substitute for its worm homologue in lifespan control; furthermore, antagonists of mammalian $\mathrm{GABA}_{\mathrm{B}}$ receptor can extend the lifespan of transgenic worms expressing this receptor, raising the possibility that $\mathrm{GABA}$ and $\mathrm{GABA}_{\mathrm{B}}$ receptor may play a role in aging in other organisms. It will be interesting to test this hypothesis in future studies.

Recent studies have uncovered an increasingly important role of the nervous system in longevity $1,3,4,7,8$. Neurons regulate lifespan in a cell-nonautonomous manner presumably through neurotransmission ${ }^{1,3,4,7,8}$. Aside from neuroendocrine signalling, neurotransmitters are also believed to play a key role in this process. For example, endoplasmic reticulum (ER) stress in neurons affects lifespan through neurotransmitter rather than neuroendocrine signalling 55 . The identity of this neurotransmitter(s), however, remains elusive. By characterizing mutant strains defective in each of the major neurotransmitters in C. elegans, we found that GABA signalling plays an important role in regulating lifespan. To the best of our knowledge, this represents the first comprehensive analysis examining the role of all neurotransmitters in lifespan control in any organism.

Our results uncovered a genetic pathway that transmits longevity signals from GABA via G protein-PLC $\beta$ signalling to 
a

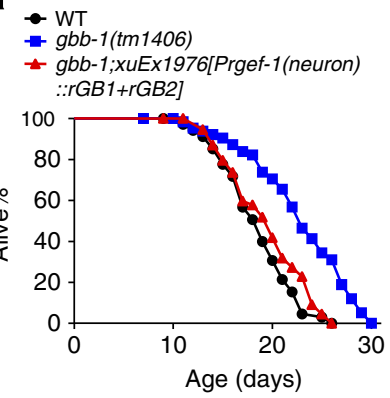

d

$\rightarrow W T$

- $g b b-1$ (tm1406)

- gbb-1(tm1406);xuEx1976

$=$ gbb-1(tm1406);xuEx1976+CGP36216

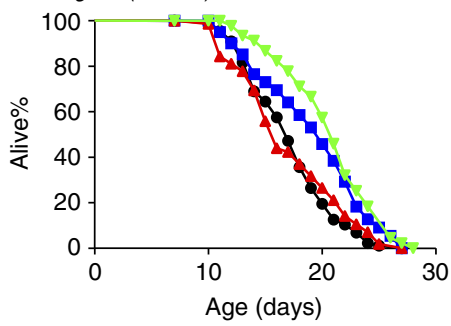

e

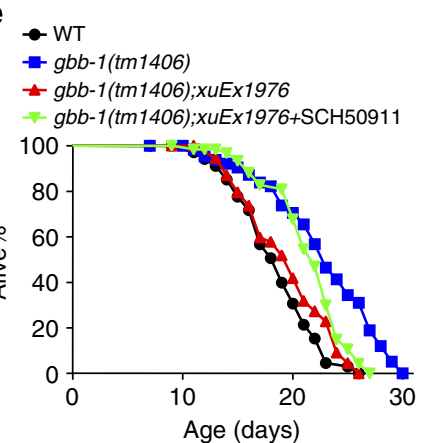

b

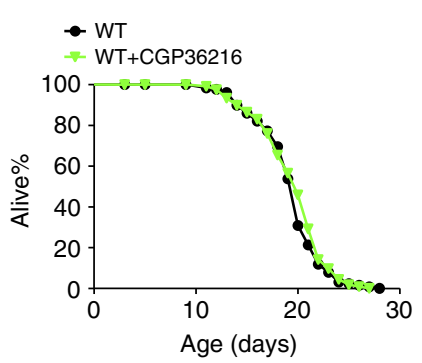

f

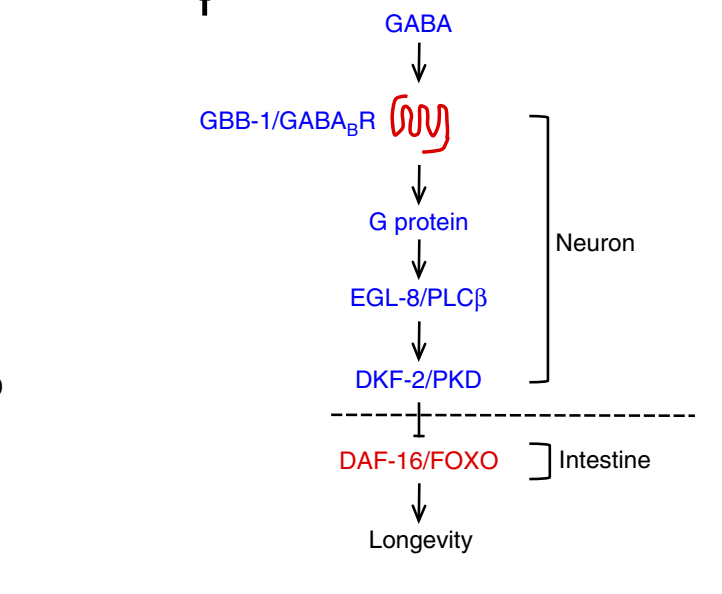

Longevity
C

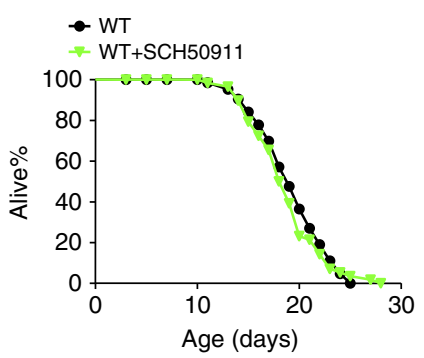

(n)

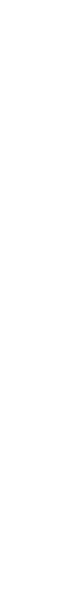


these neurotransmitters. It is also possible that these neurotransmitters may play a more prominent role in controlling lifespan under certain specific physiological conditions. Our studies will encourage others to investigate how the nervous system regulates lifespan through neurotransmitter signalling, an interesting but poorly understood question in the biology of aging.

\section{Methods}

Genetics and molecular biology. Wild-type: N2. TQ2181:unc-13(e51). TQ4927: tbh-1(n3247) $\times 8$ outcrossed. TQ4935: cat-2(e1112) $\times 8$ outcrossed. TQ4933: eat$4(k y 5) \times 8$ outcrossed. TQ4929: $t d c-1(n 3419) \times 8$ outcrossed.

TQ4931: tph-1 $(m g 280) \times 8$ outcrossed. unc-17(e245) $\times 4$ outcrossed. TQ6057: unc-25(e156) $\times 4$ outcrossed. TQ4912: $g b b-1(t m 1406) \times 8$ outcrossed. TQ4911: $g b b-2(t m 1165) \times 8$ outcrossed. TQ6054: unc-49(e407) $\times 4$ outcrossed. TQ5117 xuEx1611[Pges-1::gbb-1::SL2::mCherry]. TQ5119: xuEx1613[Pmyo-3::gbb1::SL2:::mCherry]. TQ5123: xuEx1617[Prgef-1::gbb-1::SL2:::mCherry]. TQ5842: xuEx1964[PH20::ptx + Prgef-1::DsRed]. xuEx2117[Prgef-1::gbb-1::SL2::YFP]. TQ4914: egl-8(n488) $\times 4$ outcrossed. TQ4077: daf-16(mgDf47) $\times 4$ outcrossed. TQ5450: dkf-2(pr3) $\times 6$ outcrossed. xuEx2106[Prgef-1::dkf-2::SL2::mCherry], xuEx2109[Prgef-1::dkf-2::SL2::YFP], TQ5940: xuEx1976[Prgef-1::rGB1:: SL2:: mCherry + Prgef-1::rGB2::SL2::YFP]. TQ5492: goa-1(sa734) $\times 2$ outcrossed. TQ4915: tpa-1(k530) $\times 4$ outcrossed. TQ5576: $p k c-1(n j 3) \times 4$ outcrossed. TQ5008: $d k f-1($ ok2695) $\times 4$ outcrossed. TQ5447: $d k f-1(p r 2) \times 6$ outcrossed. The $p k c-3$ RNA interference (RNAi) clone was generated in the laboratory, while other RNAi clones were from the Ahringer library and were confirmed by sequencing. All mutants in the GBB-1 pathway are null alleles, including $g b b-1, e g l-8, d k f-2$ and daf-16. These mutants have been outcrossed to our $\mathrm{N} 2$ strain for four to eight times before lifespan assay.

Microinjections were performed using standard protocols. Each plasmid DNA listed above in the transgenic line was injected at a concentration of $50 \mathrm{ng} \mu \mathrm{l}^{-1}$. For those experiments involving transgenes, three independent transgenic lines were tested for lifespan to confirm the results. For simplicity and clarity, only the data from one transgenic line were shown.

Lifespan assay. Lifespan studies were performed on 60 -mm nematode growth medium (NGM) plates at $20^{\circ} \mathrm{C}$ as previously described ${ }^{56,59,60}$. For each lifespan assay, 70-110 worms were included and transferred every other day to fresh NGM plates with 14 worms per plate. The first day of adulthood was considered day 1 . Survival was scored every 1-2 days, and worms were censored if they crawled off the plate, hatched inside or lost the vulva integrity during reproduction. 5-Fluoro- ${ }^{\prime}$-deoxyuridine was included in assays involving unc-13, unc-17 and egl-8 mutant worms, which show egg-laying defects. For RNAi experiments, NGM plates included carbenicillin $\left(25 \mu \mathrm{g} \mathrm{ml}^{-1}\right)$ and isopropyl- $\beta$-D-thiogalactoside $(1 \mathrm{mM})$. HT115 bacteria with vector or RNAi plasmid were seeded on RNAi plates 2 days before experiment. Worms were fed RNAi bacteria, beginning at the egg stage.

For $\mathrm{GABA}_{\mathrm{B}}$ receptor antagonist experiments, $1 \mu \mathrm{M}$ CGP36216 or SCH50911 (Tocris) was first spread out on NGM plates to let it diffuse for 1 day. L4 hermaphrodites were then transferred to these plates to assay lifespan. Worms were transferred to fresh drug plates every 2-3 days until day 10, after which animals remained on the same drug plates until death.

Graphpad Prism 5 (GraphPad Software Inc.) and IBM SPSS Statistics 19 (IBM Inc.) were used to analyse lifespan data. $P$ values were calculated with the log-rank (Kaplan-Meier) method.

Overexpression of rat $\mathrm{GABA}_{\mathbf{B}}$ receptor in $\mathbf{C}$. elegans. cDNA encoding the rat $\mathrm{GABA}_{B}$ receptor subunits, $\mathrm{GB} 1$ or GB2, was driven by the neuron-specific promoter rgef-1. The two plasmids were co-injected (50 $\mathrm{ng}^{-1}$ each) into $g b b-1$ mutant worms to generate the transgenic animals overexpressing rat $G_{A B A}$ receptor.

qRT-PCR and microscopy. Total RNA was isolated from $\sim 200$ Day-3 adult worms using TRI Reagent (Life Technologies). Quantitative PCR (qPCR) experiments were performed with CYBR Green (Life Technologies) according to the protocol provided by the manufacturer to analyse the amount of mRNA of daf-16 target genes. qPCR data were analysed with the $\Delta \Delta C_{\mathrm{t}}$ method using act-1 (actin) as an internal reference for normalization. Primer sequences used for qPCR are $\left(\right.$ all $\left.5^{\prime}-3^{\prime}\right)$ listed as below. act-1: $5^{\prime}$-CCAGGAATTGCTGATCGTATGCAGA A- $3^{\prime}, 5^{\prime}$-TGGAGAGGGAAGCGAGGATAGA- $3^{\prime} . m t l-1: 5^{\prime}$-TGCAGTCTCCCTTA CATCCA-3' ${ }^{\prime}$, $5^{\prime}$-TGCAGTGGAGACAAGTGTTG- ${ }^{\prime}$. sod-3: $5^{\prime}$-TATTAAGCG CGACTTCGGTTCCCT-3', 5' -CGTGCTCCCAAACGTCAATTCCAA-3' . dod-3: $5^{\prime}$-AAAAAGCCATGTTCCCGAAT-3' ${ }^{\prime}$ 5'-GCTGCGAAAAGCAAGAAAAT- ${ }^{\prime}$.

Quantification of SOD-3::GFP fluorescence intensity was performed on an Olympus BX51 upright microscope as described previously ${ }^{56}$. Images were acquired with a Roper CoolSnap charge couple device camera controlled with MetaMorph (Molecular Devices Inc.) and analysed with ImageJ (NIH). SOD3::GFP is encoded by the transgene muIs84 (ref. 46).

\section{References}

1. Kenyon, C. J. The genetics of ageing. Nature 464, 504-512 (2010).

2. Fontana, L., Partridge, L. \& Longo, V. D. Extending healthy life span--from yeast to humans. Science 328, 321-326 (2010).

3. Allen, E. N., Ren, J., Zhang, Y. \& Alcedo, J. Sensory systems: their impact on C. elegans survival. Neuroscience 296, 15-25 (2014).

4. Jeong, D. E., Artan, M., Seo, K. \& Lee, S. J. Regulation of lifespan by chemosensory and thermosensory systems: findings in invertebrates and their implications in mammalian aging. Front. Genet. 3, 218 (2012).

5. Wolkow, C. A., Kimura, K. D., Lee, M. S. \& Ruvkun, G. Regulation of C. elegans life-span by insulinlike signaling in the nervous system. Science 290, 147-150 (2000).

6. Taguchi, A., Wartschow, L. M. \& White, M. F. Brain IRS2 signaling coordinates life span and nutrient homeostasis. Science 317, 369-372 (2007).

7. Apfeld, J. \& Kenyon, C. Regulation of lifespan by sensory perception in Caenorhabditis elegans. Nature 402, 804-809 (1999).

8. Alcedo, J. \& Kenyon, C. Regulation of C. elegans longevity by specific gustatory and olfactory neurons. Neuron 41, 45-55 (2004).

9. Bettler, B., Kaupmann, K. \& Bowery, N. GABAB receptors: drugs meet clones Curr. Opin. Neurobiol. 8, 345-350 (1998).

10. Bettler, B., Kaupmann, K., Mosbacher, J. \& Gassmann, M. Molecular structure and physiological functions of GABA(B) receptors. Physiol. Rev. 84, 835-867 (2004).

11. D'Souza, M. S. \& Markou, A. The "stop" and "go" of nicotine dependence: role of GABA and glutamate. Cold Spring Harb. Perspect. Med. 3, doi:10.1101/ cshperspect.a012146 (2013).

12. Vithlani, M., Terunuma, M. \& Moss, S. J. The dynamic modulation of GABA(A) receptor trafficking and its role in regulating the plasticity of inhibitory synapses. Physiol. Rev. 91, 1009-1022 (2011).

13. McCarthy, M. M., Auger, A. P. \& Perrot-Sinal, T. S. Getting excited about GABA and sex differences in the brain. Trends Neurosci. 25, 307-312 (2002).

14. Koella, W. P. GABA systems and behavior. Adv. Biochem. Psychopharmacol. 29, 11-21 (1981).

15. Gassmann, M. \& Bettler, B. Regulation of neuronal GABA(B) receptor functions by subunit composition. Nat. Rev. Neurosci. 13, 380-394 (2012).

16. Xu, C., Zhang, W., Rondard, P., Pin, J. P. \& Liu, J. Complex GABAB receptor complexes: how to generate multiple functionally distinct units from a single receptor. Front. Pharmacol. 5, 12 (2014).

17. Bormann, J. The 'ABC' of GABA receptors. Trends Pharmacol. Sci. 21, 16-19 (2000).

18. Hosie, A. M., Aronstein, K., Sattelle, D. B. \& ffrench-Constant, R. H. Molecular biology of insect neuronal GABA receptors. Trends Neurosci. 20, 578-583 (1997).

19. Kaupmann, K. et al. Expression cloning of GABA(B) receptors uncovers similarity to metabotropic glutamate receptors. Nature 386, 239-246 (1997).

20. Jones, K. A. et al. GABA(B) receptors function as a heteromeric assembly of the subunits GABA(B)R1 and GABA(B)R2. Nature 396, 674-679 (1998).

21. Kaupmann, K. et al. GABA(B)-receptor subtypes assemble into functional heteromeric complexes. Nature 396, 683-687 (1998).

22. White, J. H. et al. Heterodimerization is required for the formation of a functional GABA(B) receptor. Nature 396, 679-682 (1998).

23. Bargmann, C. I. Neurobiology of the Caenorhabditis elegans genome. Science 282, 2028-2033 (1998)

24. de Bono, M. \& Maricq, A. V. Neuronal substrates of complex behaviors in C. elegans. Annu. Rev. Neurosci. 28, 451-501 (2005).

25. Maruyama, I. N. \& Brenner, S. A phorbol ester/diacylglycerol-binding protein encoded by the unc-13 gene of Caenorhabditis elegans. Proc. Natl Acad. Sci. USA 88, 5729-5733 (1991).

26. Richmond, J. E., Davis, W. S. \& Jorgensen, E. M. UNC-13 is required for synaptic vesicle fusion in C. elegans. Nat. Neurosci. 2, 959-964 (1999).

27. Munoz, M. J. \& Riddle, D. L. Positive selection of Caenorhabditis elegans mutants with increased stress resistance and longevity. Genetics 163, 171-180 (2003).

28. Alfonso, A., Grundahl, K., Duerr, J. S., Han, H. P. \& Rand, J. B. The Caenorhabditis elegans unc-17 gene: a putative vesicular acetylcholine transporter. Science 261, 617-619 (1993).

29. Sze, J. Y., Victor, M., Loer, C., Shi, Y. \& Ruvkun, G. Food and metabolic signalling defects in a Caenorhabditis elegans serotonin-synthesis mutant. Nature 403, 560-564 (2000).

30. Lints, R. \& Emmons, S. W. Patterning of dopaminergic neurotransmitter identity among Caenorhabditis elegans ray sensory neurons by a TGFbeta family signaling pathway and a Hox gene. Development 126, 5819-5831 (1999).

31. Alkema, M. J., Hunter-Ensor, M., Ringstad, N. \& Horvitz, H. R. Tyramine functions independently of octopamine in the Caenorhabditis elegans nervous system. Neuron 46, 247-260 (2005).

32. Lee, R. Y., Sawin, E. R., Chalfie, M., Horvitz, H. R. \& Avery, L. EAT-4, a homolog of a mammalian sodium-dependent inorganic phosphate 
cotransporter, is necessary for glutamatergic neurotransmission in Caenorhabditis elegans. J. Neurosci. 19, 159-167 (1999).

33. Jin, Y., Jorgensen, E., Hartwieg, E. \& Horvitz, H. R. The Caenorhabditis elegans gene unc-25 encodes glutamic acid decarboxylase and is required for synaptic transmission but not synaptic development. J. Neurosci. 19, 539-548 (1999).

34. Dittman, J. S. \& Kaplan, J. M. Behavioral impact of neurotransmitter-activated G-protein-coupled receptors: muscarinic and $\mathrm{GABAB}$ receptors regulate Caenorhabditis elegans locomotion. J. Neurosci 28, 7104-7112 (2008).

35. Bamber, B. A., Beg, A. A., Twyman, R. E. \& Jorgensen, E. M. The Caenorhabditis elegans unc- 49 locus encodes multiple subunits of a heteromultimeric GABA receptor. J. Neurosci. 19, 5348-5359 (1999).

36. Kimura, K. D., Tissenbaum, H. A., Liu, Y. \& Ruvkun, G. daf-2, an insulin receptor-like gene that regulates longevity and diapause in Caenorhabditis elegans. Science 277, 942-946 (1997).

37. $\mathrm{Tu}, \mathrm{H}$. et al. GABAB receptor activation protects neurons from apoptosis via IGF-1 receptor transactivation. J. Neurosci. 30, 749-759 (2010).

38. Mendel, J. E. et al. Participation of the protein Go in multiple aspects of behavior in C. elegans. Science 267, 1652-1655 (1995).

39. Segalat, L., Elkes, D. A. \& Kaplan, J. M. Modulation of serotonin-controlled behaviors by Go in Caenorhabditis elegans. Science 267, 1648-1651 (1995).

40. Roayaie, K., Crump, J. G., Sagasti, A. \& Bargmann, C. I. The G alpha protein ODR-3 mediates olfactory and nociceptive function and controls cilium morphogenesis in C. elegans olfactory neurons. Neuron 20, 55-67 (1998).

41. Lackner, M. R., Nurrish, S. J. \& Kaplan, J. M. Facilitation of synaptic transmission by EGL-30 Gqalpha and EGL-8 PLCbeta: DAG binding to UNC13 is required to stimulate acetylcholine release. Neuron 24, 335-346 (1999).

42. Miller, K. G., Emerson, M. D. \& Rand, J. B. Goalpha and diacylglycerol kinase negatively regulate the Gqalpha pathway in C. elegans. Neuron 24, 323-333 (1999).

43. Ch'ng, Q., Sieburth, D. \& Kaplan, J. M. Profiling synaptic proteins identifies regulators of insulin secretion and lifespan. PLoS Genet. 4, e1000283 (2008).

44. McKinsey, T. A. Derepression of pathological cardiac genes by members of the CaM kinase superfamily. Cardiovasc. Res. 73, 667-677 (2007).

45. Feng, H., Ren, M., Chen, L. \& Rubin, C. S. Properties, regulation, and in vivo functions of a novel protein kinase D: Caenorhabditis elegans DKF-2 links diacylglycerol second messenger to the regulation of stress responses and life span. J. Biol. Chem. 282, 31273-31288 (2007).

46. Libina, N., Berman, J. R. \& Kenyon, C. Tissue-specific activities of C. elegans DAF-16 in the regulation of lifespan. Cell 115, 489-502 (2003).

47. Rondard, P. et al. Functioning of the dimeric GABA(B) receptor extracellular domain revealed by glycan wedge scanning. EMBO J. 27, 1321-1332 (2008).

48. Galvez, T. et al. Allosteric interactions between GB1 and GB2 subunits are required for optimal GABA(B) receptor function. EMBO J. 20, 2152-2159 (2001).

49. Pin, J. P. et al. Activation mechanism of the heterodimeric GABA(B) receptor. Biochem. Pharmacol. 68, 1565-1572 (2004).

50. Couve, A., Moss, S. J. \& Pangalos, M. N. GABAB receptors: a new paradigm in G protein signaling. Mol. Cell Neurosci. 16, 296-312 (2000).

51. Comps-Agrar, L. et al. The oligomeric state sets GABA(B) receptor signalling efficacy. EMBO J. 30, 2336-2349 (2011).

52. Ong, J. et al. CGP 36216 is a selective antagonist at GABA(B) presynaptic receptors in rat brain. Eur. J. Pharmacol. 415, 191-195 (2001).

53. Froestl, W. Chemistry and pharmacology of GABAB receptor ligands. $A d v$. Pharmacol. 58, 19-62 (2010).

54. Enell, L. E., Kapan, N., Soderberg, J. A., Kahsai, L. \& Nassel, D. R. Insulin signaling, lifespan and stress resistance are modulated by metabotropic GABA receptors on insulin producing cells in the brain of Drosophila. PLOS ONE 5, e15780 (2010)

55. Taylor, R. C. \& Dillin, A. XBP-1 is a cell-nonautonomous regulator of stress resistance and longevity. Cell 153, 1435-1447 (2013).
56. Xiao, R. et al. A genetic program promotes C. elegans longevity at cold temperatures via a thermosensitive TRP channel. Cell 152, 806-817 (2013).

57. Burkewitz, K. et al. Neuronal CRTC-1 governs systemic mitochondrial metabolism and lifespan via a catecholamine signal. Cell 160, 842-855 (2015)

58. Yin, J. A., Liu, X. J., Yuan, J., Jiang, J. \& Cai, S. Q. Longevity manipulations differentially affect serotonin/dopamine level and behavioral deterioration in aging Caenorhabditis elegans. J. Neurosci. 34, 3947-3958 (2014).

59. Hsu, A. L., Feng, Z., Hsieh, M. Y. \& Xu, X. Z. Identification by machine vision of the rate of motor activity decline as a lifespan predictor in C. elegans. Neurobiol. Aging 30, 1498-1503 (2009).

60. Liu, J. et al. Functional aging in the nervous system contributes to age-dependent motor activity decline in C. elegans. Cell Metab. 18, 392-402 (2013).

61. Aamodt, E. J., Chung, M. A. \& McGhee, J. D. Spatial control of gut-specific gene expression during Caenorhabditis elegans development. Science 252, 579-582 (1991)

62. Altun-Gultekin, Z. et al. A regulatory cascade of three homeobox genes, ceh-10 ttx-3 and ceh-23, controls cell fate specification of a defined interneuron class in C. elegans. Development 128, 1951-1969 (2001).

63. Fire, A. \& Waterston, R. H. Proper expression of myosin genes in transgenic nematodes. EMBO J. 8, 3419-3428 (1989).

64. Cao, P. et al. Light-sensitive coupling of rhodopsin and melanopsin to G(i/o) and G(q) signal transduction in Caenorhabditis elegans. FASEB J. 26, 480-491 (2012).

\section{Acknowledgements}

We thank Charles Rubin and Ao-Lin Hsu for providing strains, Zhaoyang Feng for the PTX plasmid and Lingxiu Xu for technical assistance. Some strains were obtained from the CGC and Knockout Consortiums in the USA and Japan. This work was supported by the NSFC (31130028, 31225011 and 31420103909 to J.L.), the Program of Introducing Talents of Discipline to the Universities from the Ministry of Education (B08029 to J.L.) the Ministry of Science and Technology of China (2012CB51800 to J.L.), Natural Science Foundation of HuBei Province (2014CFA010 to J.L.) and the NIA (X.Z.S.X.).

\section{Author contributions}

L.C. performed the experiments and analysed the data. J.G. and B.Z. initiated the project J.G., F.Y., H.L., T.Z. and T.Y. performed some experiments. X.Z.S.X. and J.L. supervised the project. C.L., X.Z.S.X. and J.L. wrote the manuscript.

\section{Additional information}

Supplementary Information accompanies this paper at http://www.nature.com/ naturecommunications

Competing financial interests: The authors declare no competing financial interests.

Reprints and permission information is available online at http://npg.nature.com/ reprintsandpermissions/

How to cite this article: Chun, L. et al. Metabotropic GABA signalling modulates longevity in C. elegans. Nat. Commun. 6:8828 doi: 10.1038/ncomms9828 (2015).

This work is licensed under a Creative Commons Attribution 4.0 International License. The images or other third party material in this article are included in the article's Creative Commons license, unless indicated otherwise in the credit line; if the material is not included under the Creative Commons license, users will need to obtain permission from the license holder to reproduce the material To view a copy of this license, visit http://creativecommons.org/licenses/by/4.0/ 